\title{
The Relationship between Obstructive Sleep Apnea and Functional Dyspepsia
}

\author{
Dae Lim Koo, Hyunwoo Nam \\ Department of Neurology, Boramae Medical Center, Seoul National University College of Medicine, Seoul, Korea
}

Received April 18, 2020 Revised May 23, 2020 Accepted June 4, 2020

Address for correspondence Hyunwoo Nam, MD, PhD

Department of Neurology, Boramae Medical Center, Seoul National University College of Medicine, 20 Boramae-ro 5-gil, Dongjak-gu, Seoul 07061, Korea

Tel: $+82-2-870-2471$

Fax: +82-2-831-3866

E-mail: hwnam85@gmail.com
Objectives: Functional dyspepsia is one of the most common functional gastrointestinal disorders. We aimed to investigate the relationship between obstructive sleep apnea (OSA) and functional dyspepsia in patients with the complaint of sleep-disordered breathing. Methods: We prospectively recruited patients who visited the institute for the evaluation of sleep-disordered breathing. All patients underwent overnight polysomnography and submitted their responses to sleep questionnaires. A validated Korean version of the Rome III criteria was used to estimate functional dyspepsia. Functional dyspepsia was classified into two subtypes of epigastric pain syndrome and postprandial distress syndrome. Results: Out of 130 subjects with sleep disturbances, a total of 79 patients ( 60 men, 19 women) were enrolled. The mean ( \pm standard deviation) age was $46.9 \pm 13.4$ years. Functional dyspepsia was diagnosed in $8(10 \%)$ patients. Patients with functional dyspepsia showed significantly higher values of Stanford Sleepiness Scale, the proportion of N1 sleep, arousal index, and apnea-hypopnea index compared to patients without functional dyspepsia ( $p=0.018, p=0.021, p=0.041$, and $p=0.039$, respectively). With respect to OSA severity, $12 \%$ of patients with moderate OSA and $22 \%$ patients with severe OSA had functional dyspepsia. Severe OSA was significantly associated with a higher proportion of postprandial distress syndrome $(p=0.030)$. Conclusions: Our patients with snoring or OSA revealed that functional dyspepsia is associated with more severe daytime sleepiness and apnea-hypopnea index compared to those without functional dyspepsia. In addition, postprandial distress syndrome was a prevalent subtype of functional dyspepsia in patients with severe OSA.

J Sleep Med 2020;17(1):73-77

Key Words: Sleep, Obstructive sleep apnea, Dyspepsia, Gastrointestinal disorder.

\section{Introduction}

Functional dyspepsia, a disorder of the upper gastrointestinal tract affecting $5-20 \%$ of the worldwide population, is one of the most common functional gastrointestinal disorders. ${ }^{1-4}$ Functional dyspepsia is generally assessed using the Rome III symptom-based diagnostic criteria published by the Rome foundation. These criteria have played an important role in the research and diagnosis of functional gastrointestinal disorders because of a current lack of diagnostic biological markers. ${ }^{5-7}$ Functional dyspepsia has been considered to provoke disturbances of gastric physiologic factors, such as slow gastric emptying, failure of the gastric fundus to relax after a meal, and gastric hypersensitivity with distention of the stomach. ${ }^{1}$ Psy-

This is an Open Access article distributed under the terms of the Creative Commons Attribution Non-Commercial License (https://creativecommons.org/licenses/by-nc/4.0) which permits unrestricted non-commercial use, distribution, and reproduction in any medium, provided the original work is properly cited. chological stress, infection, and inflammation could be candidates that cause functional dyspepsia; however, its pathomechanism remains unclear. ${ }^{8,9}$

Obstructive sleep apnea (OSA) is characterized by complete or partial obstruction of the upper airway. Recurrent hypoxic or apneic episodes cause intermittent hypoxemia, hypercapnia, microarousals, and fragmented sleep. ${ }^{10,11}$ OSA has been associated with increased risk of hypertension, type 2 diabetes mellitus, and with various cardiovascular and cerebrovascular disease risk factors. ${ }^{12-15}$ Gastroesophageal reflux has been associated with OSA in the previous studies. ${ }^{16,17}$ It is also well known that sleep disturbances are linked to functional gastrointestinal disorders and altered intestinal sensitivity. A previous report showed that poor sleep quality is associated with functional gastrointestinal symptoms in the general population. ${ }^{6}$ Insufficient sleep duration with reduced sleep quality might be a causal factor that results in emotional, cognitive, and 
somatic effects. ${ }^{18}$ Altered sleep duration and impaired sleep quality are common night symptoms in patients with OSA.

In the current study, we aimed to investigate the prevalence of functional dyspepsia in patients with untreated OSA. Clinical and polysomnographic parameters correlated with functional dyspepsia were assessed in patients with the complaint of sleep disordered breathing.

\section{Methods}

\section{Study participants}

Individuals with complaints of snoring or sleep-disordered breathing were prospectively screened at the Boramae Medical Center of Seoul National University between January 2018 and June 2019. One hundred-thirty subjects with sleep disturbances were initially screened. Each patient had a detailed clinical interview and submitted responses to a sleep questionnaire. Thirty-four patients were excluded according to the following criteria (Fig. 1): 1) insomnia ( $\mathrm{n}=9), 2)$ parasomnia ( $\mathrm{n}=$ 18), 3) excessive daytime sleepiness ( $n=2)$, and 4 ) restless leg syndrome or periodic limb movement disorder $(n=5)$. Overnight polysomnography was applied to 96 patients with night symptoms, including snoring, shortness of breath, or witnessed apnea during sleep. Seventeen patients with a greater than 15 per hour of periodic limb movement during sleep index were excluded. Finally, a total of 79 patients were enrolled in this study. Approval for this study was obtained from the Institutional Review Board at the Boramae Medical Center of Seoul National University. Written informed consent for participation in this study was obtained from each patient or his/her legal representative.

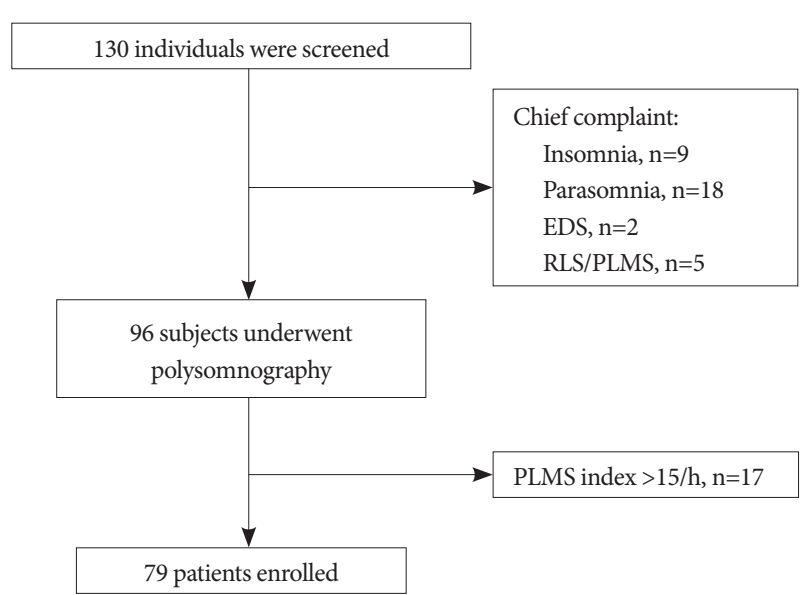

Figure 1. Study flow diagram. Out of 130 individuals with sleep disturbances, 79 patients were included in this study. EDS: excessive daytime sleepiness, RLS: restless leg syndrome, PLMS: periodic limb movement during sleep.

\section{Sleep questionnaires and polysomnography}

Subjective daytime sleepiness was estimated by applying the Epworth Sleepiness Scale and the Stanford Sleepiness Scale. The Pittsburgh sleep quality index (PSQI) was used to estimate the sleep disturbances and quality during the last month wherein a total score of higher than 5 indicated poor sleep quality. ${ }^{19}$ Patients were instructed not to ingest alcohol or caffeinated beverages and to sleep and wake up at their regular hours for one week before the study. Polysomnography data were recorded using the Twin-polysomnography software (Natus Neurology Incorporated, West Warwick, RI, USA), a 6-channel electroencephalogram, a 4-channel electrooculogram, an electromyogram, and an electrocardiogram. A thermistor, a nasal air pressure monitoring sensor, an oximeter, piezoelectric bands, and a body position sensor were also connected to the patient. Obstructive apnea was defined as an airflow reduction of $90 \%$ or more lasting at least $10 \mathrm{~s}$. Hypopnea was defined as an airflow reduction of more than $30 \%$, with concurrent reduction in oxyhemoglobin saturation by at least $3 \%$, or arousals from sleep. ${ }^{11}$ OSA was graded according to the apnea-hypopnea index (AHI): AHI $<5$ (events/h) was considered simple snoring without sleep apnea, $5 \leq \mathrm{AHI}<15$ was considered mild OSA, $15 \leq \mathrm{AHI}<30$ was considered moderate OSA, and $\mathrm{AHI} \geq 30$ was considered severe OSA.

\section{Evaluation of functional dyspepsia}

A validated Korean version of the Rome III criteria was used for the assessment of functional dyspepsia. ${ }^{20}$ Patients were diagnosed with functional dyspepsia if they had been experiencing one or more of the following symptoms for at least 3 months with symptom onset in at least the previous 6 months: bothersome postprandial fullness, early satiation, epigastric pain, and/or epigastric burning, with no evidence of structural disease. Functional dyspepsia was classified into epigastric pain syndrome and postprandial distress syndrome subtypes. ${ }^{1}$

\section{Statistical analysis}

The participants in this study were divided into two groups based on the presence of functional dyspepsia. To compare the clinical and polysomnographic parameters between the groups, we used t-tests for continuous variables and $\chi^{2}$ or Fisher's exact tests for categorical variables in this analysis. All the continuous quantitative parameters are represented as mean \pm standard deviation. In addition, our subjects were divided 3 groups according to the severity of OSA. Differences in variables among these subgroups were measured by $\chi^{2}$ or Fisher's exact tests with adjustments for multiple comparisons using a Bonferroni correction. Two-sided $p$-values less than 0.05 were considered statistically significant. Statistical analyses were performed using SPSS statistical software version 26 (IBM 
Corp., Armonk, NY, USA).

\section{Results}

The patients' age and body mass index (BMI) were $46.9 \pm 13.4$ years and $25.5 \pm 3.8 \mathrm{~kg} / \mathrm{m}^{2}$, respectively. Of the 79 patients, 19 (24\%) were women. Eight (10\%) with complaints of snoring or sleep apnea showed functional dyspepsia. Patients with functional dyspepsia showed a significant daytime sleepiness with higher scores of Stanford Sleepiness Scale $(p=0.018)$. The PSQI score of patients with functional dyspepsia was $11.0 \pm 3.5$, which was significantly higher than $8.1 \pm 3.0$ of the PSQI in patients without functional dyspepsia ( $p=0.036$ ). Among the polysomnographic parameters, the proportion of N1 sleep and rapid eye movement sleep latency were significantly increased in pa- tients with functional dyspepsia ( $p=0.021$ and $p=0.044$, respectively). Functional dyspepsia group showed a significantly higher values of arousal index and AHI compared to patients without functional dyspepsia ( $p=0.041$ and $p=0.039$, respectively). The detailed clinical demographics and polysomnographic findings of each group are summarized in Table 1. Of the total 79 subjects, 66 (84\%) patients were diagnosed as OSA with $\mathrm{AHI} \geq 5 / \mathrm{h}$. The prevalence of functional dyspepsia in patients with OSA was $12 \%$, which was higher than that in patients with simple snoring $(0 \%)$, but this difference was not significant (Table 2). OSA group showed significantly higher age and increased BMI compared to simple snoring group $(p=$ 0.003 and $p=0.038$ ). Regarding the severity of OSA, $12 \%$ of patients with moderate OSA and 22\% patients with severe OSA had functional dyspepsia; in patients with mild OSA, $0 \%$ had

Table 1. Clinical and polysomnographic characteristics between SDB groups with or without functional dyspepsia

\begin{tabular}{|c|c|c|c|}
\hline & SDB patients with FD & SDB patients without FD & $p$ \\
\hline \multicolumn{4}{|l|}{ Clinical factors } \\
\hline Subjects, $\mathrm{n}$ & 8 & 71 & - \\
\hline Age, years, mean (SD) & $43.4(15.7)$ & $47.3(13.2)$ & 0.425 \\
\hline Men, n (\%) & $7(87.5)$ & $53(74.6)$ & 0.423 \\
\hline BMI, kg/m², mean (SD) & $28.3(4.4)$ & $25.5(3.7)$ & 0.079 \\
\hline ESS score, mean (SD) & $11.0(5.2)$ & $7.7(4.7)$ & 0.118 \\
\hline SSS score, mean (SD) & $3.3(0.5)$ & $2.6(0.9)$ & 0.018 \\
\hline PSQI score, mean (SD) & $11.0(3.5)$ & $8.1(3.0)$ & 0.036 \\
\hline Hypertension, $\mathrm{n}(\%)$ & $2(25.0)$ & $13(18.3)$ & 0.649 \\
\hline Diabetes mellitus, n (\%) & $1(12.5)$ & $2(2.8)$ & 0.177 \\
\hline Smoking, n (\%) & $3(37.5)$ & $16(22.5)$ & 0.235 \\
\hline Alcohol, n (\%) & $3(37.5)$ & $39(54.9)$ & 0.544 \\
\hline \multicolumn{4}{|l|}{ Polysomnographic parameters } \\
\hline Time in bed, min, mean (SD) & $410.0(34.2)$ & $412.8(56.4)$ & 0.782 \\
\hline Total sleep time, min, mean (SD) & $311.3(43.2)$ & $339.4(60.6)$ & 0.083 \\
\hline N1 sleep, \%, mean (SD) & $24.5(4.9)$ & $18.4(10.3)$ & 0.021 \\
\hline $\mathrm{N} 2$ sleep, $\%$, mean $(\mathrm{SD})$ & $41.5(9.0)$ & $45.6(9.3)$ & 0.249 \\
\hline N3 sleep, \%, mean (SD) & $16.8(5.9)$ & $17.3(7.6)$ & 0.820 \\
\hline REM sleep, \%, mean (SD) & $17.2(7.7)$ & $20.8(15.3)$ & 0.354 \\
\hline Sleep latency, min, mean (SD) & $14.3(13.9)$ & $10.9(19.1)$ & 0.349 \\
\hline REM sleep latency, min, mean (SD) & $161.8(59.1)$ & $121.3(65.2)$ & 0.044 \\
\hline Sleep efficiency, \%, mean (SD) & $77.1(8.9)$ & $81.8(13.7)$ & 0.081 \\
\hline Arousal index, events/h, mean (SD) & $28.5(7.3)$ & $22.7(13.8)$ & 0.041 \\
\hline PLMS index, events/h, mean (SD) & $0.1(0.3)$ & $1.2(3.2)$ & 0.742 \\
\hline AHI, events/h, mean (SD) & $32.8(15.5)$ & $21.4(17.3)$ & 0.039 \\
\hline Longest sleep apnea, sec, mean (SD) & $29.4(15.7)$ & $25.4(24.7)$ & 0.424 \\
\hline Lowest oxygen saturation, \%, mean (SD) & $78.8(8.7)$ & $80.0(12.4)$ & 0.454 \\
\hline
\end{tabular}

SDB: sleep-disordered breathing, FD: functional dyspepsia, SD: standard deviation, BMI: body mass index, ESS: Epworth Sleepiness Scale, SSS: Stanford Sleepiness Scale, PSQI: Pittsburgh sleep quality index, REM: rapid eye movement, PLMS: periodic limb movement during sleep, AHI: apnea-hypopnea index 
Table 2. Difference between patients with and without OSA

\begin{tabular}{lccc}
\hline & $\begin{array}{c}\text { Simple snoring } \\
\text { group }\end{array}$ & OSA group & $p$ \\
\hline Subjects, n (\%) & $13(16.5)$ & $66(83.5)$ & - \\
Age, years, mean (SD) & $37.8(11.8)$ & $48.7(13.0)$ & 0.003 \\
Men, n (\%) & $9(69.2)$ & $35(77.3)$ & 0.538 \\
BMI, kg/m², mean (SD) & $23.7(2.6)$ & $26.1(3.9)$ & 0.038 \\
ESS score, mean (SD) & $7.0(4.2)$ & $8.2(5.0)$ & 0.431 \\
SSS score, mean (SD) & $2.5(1.0)$ & $2.7(0.9)$ & 0.658 \\
PSQI score, mean (SD) & $8.6(2.7)$ & $8.3(3.3)$ & 0.593 \\
Smoking, n (\%) & $2(15.4)$ & $17(25.8)$ & 0.412 \\
Alcohol, n (\%) & $10(76.9)$ & $32(48.5)$ & 0.069 \\
Functional dyspepsia, n (\%) & $0(0)$ & $8(12.1)$ & 0.188 \\
EPS & 0 & 4 & - \\
PDS & 0 & 2 & - \\
Both EPS and PDS & 0 & 2 & -
\end{tabular}

OSA: obstructive sleep apnea, SD: standard deviation, BMI: body mass index, ESS: Epworth Sleepiness Scale, SSS: Stanford Sleepiness Scale, PSQI: Pittsburgh sleep quality index, EPS: epigastric pain syndromes, PDS: postprandial distress syndromes

Table 3. Prevalence of FD according to the severity of OSA

\begin{tabular}{lcccc}
\hline & \multicolumn{3}{c}{ Severity of OSA } & \multirow{2}{*}{$p^{*}$} \\
\cline { 2 - 4 } & $\begin{array}{c}(1) \text { Mild }(2) \text { Moderate }(3) \text { Severe } \\
(\mathrm{n}=17)\end{array}$ & $(\mathrm{n}=26)$ & $(\mathrm{n}=23)$ & \\
\hline FD, n (\%) & $0(0)$ & $3(12)$ & $5(22)$ & 0.117 \\
EPS & $0(0)$ & $3(12)$ & $1(4)$ & 0.323 \\
PDS & $0(0)$ & $0(0)$ & $2(9)$ & 0.017 \\
& & & & $(1)$ vs. $(3) ; 0.062$ \\
& & & & $(2)$ vs. $(3) ; 0.030$ \\
& & & & $(1)$ vs. $(2) ; 1.000$ \\
EPS and PDS & $0(0)$ & $0(0)$ & $2(9)$ & 0.151 \\
\hline
\end{tabular}

${ }^{*} p$ value from Fisher's exact tests with Bonferroni multiple testing correction among groups (1), (2), and (3). FD: functional dyspepsia, OSA: obstructive sleep apnea, EPS: epigastric pain syndromes, PDS: postprandial distress syndromes

functional dyspepsia (Table 3). Although the prevalence of functional dyspepsia showed no significant difference among three OSA subgroups, severe OSA group was significantly associated with a higher proportion of postprandial distress syndrome compared to moderate OSA group in the subtype analyses of functional dyspepsia $(p=0.030)$.

\section{Discussion}

This prospective observational study has demonstrated that patients with functional dyspepsia showed significantly higher AHI compared to the other group without functional dyspepsia. Seep quality was much more impaired in OSA patients with functional dyspepsia than those without functional dyspepsia. Patients with OSA $(\mathrm{AHI} \geq 5 / \mathrm{h})$ revealed a higher proportion of functional dyspepsia in comparison with that in simple snoring group. Furthermore, severe degree of OSA might be associated with postprandial distress syndrome subtype of functional dyspepsia. To the best of our knowledge, this is the first report to demonstrate the association between sleep apnea and functional dyspepsia in patients with sleep-disordered breathing.

Sleep disturbance and gastrointestinal symptoms have been considered as independent predictors for impaired quality of life after adjusting for age, gender, and BMI in a previous population-based study, ${ }^{6}$ where it was reported that poor sleep was associated with increased odds for multiple upper and lower gastrointestinal symptoms. In another study of 212 patients from a gastrointestinal clinic, the PSQI and Insomnia Severity Index were the parameters used. ${ }^{21}$ Irritable bowel syndrome group had the highest frequency of poor sleep (72\%) and clinical insomnia (51\%) compared to those in the celiac disease, gastroesophageal reflux disease, inflammatory bowel disease, and healthy control groups; functional dyspepsia was not included in that study due to the small sample size. A recent study which considered the health checkup data of 2,674 participants showed that $36 \%$ had sleep disturbance, which was associated with higher prevalence of functional dyspepsia. ${ }^{22}$ However, most of previous studies were solely based on subjective diagnostic tools including questionnaires on sleep disturbance and functional dyspepsia.

In this study, overnight polysomnography, which is a standard and objective tool for the diagnosis of OSA, was applied to elucidate the relationship between OSA and functional dyspepsia. OSA patients comorbid with functional dyspepsia showed more severe statuses of sleep apnea and respiratory arousals during night than those without functional dyspepsia. In addition, OSA group revealed a higher proportion of functional dyspepsia than simple snoring group, but not significant. In the aspects of cardiovascular pathogenesis of OSA, systemic inflammatory process has been considered to play a central. ${ }^{23}$ The repetitive cycles of short periods of hypoxia followed by rapid reoxygenation could activate inflammatory pathways. Interestingly, inflammation is also one of pathophysiological mechanisms in functional dyspepsia. ${ }^{9}$ We propose that inflammatory process might be a possible common pathophysiology for the enhanced association between OSA and functional dyspepsia. In our additional analyses for the subtypes of functional dyspepsia, postprandial distress syndrome was significantly associated with severe OSA in comparison with moderate OSA group. In the previous studies, the innate inflammation, which is characterized by an eosinophil infiltrate in the duodenum, has been considered to the inflamma- 
tory phenotype of functional dyspepsia in patients with postprandial distress syndrome..$^{24,25}$

Several limitations should be considered when interpreting the results of the current study. First, the number of positive samples for functional dyspepsia was very small, which made it difficult to identify the reliable outcomes of this study. Second, the small sample in each OSA group and multiple comparisons by OSA severity reduced the statistical power of the study. Finally, age and BMI had not been included as covariates in our analyses for the relationship between OSA and functional dyspepsia although older age and higher BMI are risk factors for OSA.

In conclusion, our patients with snoring or OSA showed that functional dyspepsia is associated with more severe daytime sleepiness and AHI compared to those without functional dyspepsia. Furthermore, postprandial distress syndrome was significantly linked to severe OSA compared to moderate OSA. Following studies with larger sample sizes need to be performed to further elucidate the relationship between OSA and functional dyspepsia.

\section{Acknowledgments}

The current study was supported by the Research Grant of Korean Sleep Research Society in 2018.

\section{Conflicts of Interest}

The authors have no potential conflicts of interest to disclose.

\section{ORCID iDs}

Dae Lim Koo

Hyunwoo Nam

https://orcid.org/0000-0001-6858-6093

https://orcid.org/0000-0003-3345-7069

\section{Author Contributions}

Conceptualization: Dae Lim Koo, Hyunwoo Nam. Data curation: Dae Lim Koo. Formal analysis: Dae Lim Koo, Hyunwoo Nam. Methodology: Dae Lim Koo, Hyunwoo Nam. Supervision: Hyunwoo Nam. Writingoriginal draft: Dae Lim Koo. Writing—review \& editing: Hyunwoo Nam.

\section{REFERENCES}

1. Talley NJ, Ford AC. Functional Dyspepsia. N Engl J Med 2015;373: 1853-1863.

2. Masuy I, Van Oudenhove L, Tack J. Review article: treatment options for functional dyspepsia. Aliment Pharmacol Ther 2019;49:1134-1172.

3. Drossman DA. Functional gastrointestinal disorders: history, pathophysiology, clinical features and Rome IV. Gastroenterology 2016;150:12621279.e2.

4. Vege SS, Locke GR 3rd, Weaver AL, Farmer SA, Melton LJ 3rd, Talley NJ. Functional gastrointestinal disorders among people with sleep disturbances: a population-based study. Mayo Clin Proc 2004;79:1501-1506.

5. Ford AC, Bercik P, Morgan DG, Bolino C, Pintos-Sanchez MI, Moayye- di P. The Rome III criteria for the diagnosis of functional dyspepsia in secondary care are not superior to previous definitions. Gastroenterology 2014;146:932-940; quiz e14-e15.

6. Cremonini F, Camilleri M, Zinsmeister AR, Herrick LM, Beebe T, Talley NJ. Sleep disturbances are linked to both upper and lower gastrointestinal symptoms in the general population. Neurogastroenterol Motil 2009;21:128-135.

7. Park JH, Jung YM, Lee HJ, Seo JY. Prevalence and factors related to irritable bowel syndrome in university students. J Korean Acad Fundam Nurs 2018;25:282-292.

8. Lim SK, Yoo SJ, Koo DL, et al. Stress and sleep quality in doctors working on-call shifts are associated with functional gastrointestinal disorders. World J Gastroenterol 2017;23:3330-3337.

9. Brun R, Kuo B. Functional dyspepsia. Therap Adv Gastroenterol 2010; 3:145-164.

10. Young T, Palta M, Dempsey J, Skatrud J, Weber S, Badr S. The occurrence of sleep-disordered breathing among middle-aged adults. $N$ Engl J Med 1993;328:1230-1235.

11. Ruehland WR, Rochford PD, O'Donoghue FJ, Pierce RJ, Singh P, Thornton AT. The new AASM criteria for scoring hypopneas: impact on the apnea hypopnea index. Sleep 2009;32:150-157.

12. Koo DL, Nam H, Thomas RJ, Yun CH. Sleep disturbances as a risk factor for stroke. J Stroke 2018;20:12-32.

13. Mashaqi S, Gozal D. Obstructive sleep apnea and systemic hypertension: gut dysbiosis as the mediator? J Clin Sleep Med 2019;15:1517-1527.

14. Fallahi A, Jamil DI, Karimi EB, Baghi V, Gheshlagh RG. Prevalence of obstructive sleep apnea in patients with type 2 diabetes: a systematic review and meta-analysis. Diabetes Metab Syndr 2019;13:2463-2468.

15. Barros D, García-Río F. Obstructive sleep apnea and dyslipidemia: from animal models to clinical evidence. Sleep 2019;42:zsy236.

16. Yang YX, Spencer G, Schutte-Rodin S, Brensinger C, Metz DC. Gastroesophageal reflux and sleep events in obstructive sleep apnea. Eur J Gastroenterol Hepatol 2013;25:1017-1023.

17. Vela MF, Kramer JR, Richardson PA, Dodge R, El-Serag HB. Poor sleep quality and obstructive sleep apnea in patients with GERD and Barrett's esophagus. Neurogastroenterol Motil 2014;26:346-352.

18. Roehrs T, Hyde M, Blaisdell B, Greenwald M, Roth T. Sleep loss and REM sleep loss are hyperalgesic. Sleep 2006;29:145-151.

19. Buysse DJ, Reynolds CF 3rd, Monk TH, Berman SR, Kupfer DJ. The Pittsburgh sleep quality index: a new instrument for psychiatric practice and research. Psychiatry Res 1989;28:193-213.

20. Song KH, Jung HK, Min BH, et al. Development and validation of the Korean Rome III Questionnaire for diagnosis of functional gastrointestinal disorders. J Neurogastroenterol Motil 2013;19:509-515.

21. Ballou S, Alhassan E, Hon E, et al. Sleep disturbances are commonly reported among patients presenting to a gastroenterology clinic. Dig Dis Sci 2018;63:2983-2991.

22. Lei WY, Chang WC, Wong MW, et al. Sleep disturbance and its association with gastrointestinal symptoms/diseases and psychological comorbidity. Digestion 2019;99:205-212.

23. Ryan S, Taylor CT, McNicholas WT. Systemic inflammation: a key factor in the pathogenesis of cardiovascular complications in obstructive sleep apnoea syndrome? Thorax 2009;64:631-636.

24. Walker MM, Salehian SS, Murray CE, et al. Implications of eosinophilia in the normal duodenal biopsy-an association with allergy and functional dyspepsia. Aliment Pharmacol Ther 2010;31:1229-1236.

25. Walker MM, Talley NJ. The role of duodenal inflammation in functional dyspepsia. J Clin Gastroenterol 2017;51:12-18. 